\title{
ON EINSTEIN MATSUMOTO METRICS
}

\author{
YI-BING SHEN AND XIAOLING ZHANG
}

\begin{abstract}
In this paper, the necessary and sufficient conditions for Matsumoto metrics $F=\frac{\alpha^{2}}{\alpha-\beta}$ to be Einstein are given. It is shown that if the length of $\beta$ with respect to $\alpha$ is constant, then the Matsumoto metric $F$ is an Einstein metric if and only if $\alpha$ is Ricci-flat and $\beta$ is parallel with respect to $\alpha$. A nontrivial example of Ricci flat Matsumoto metrics is given.
\end{abstract}

\section{INTRODUCTION}

Let $F=F(x, y)$ be a Finsler metric on an n-dimensional manifold $M . F$ is called an Einstein metric with Einstein scalar $\sigma$ if its Ricci curvature Ric satisfies

$$
\operatorname{Ric}=\sigma F^{2},
$$

where $\sigma=\sigma(x)$ is a scalar function on $M$. In particular, $F$ is said to be Ricci constant (resp. Ricci flat) if $\sigma=$ const. (resp. $\sigma=0)$ in (1.1). ([2], [3]).

An important class of Finsler metrics is so called $(\alpha, \beta)$-metrics, which are iteratively appearing in physical studies, and are expressed in the form of $F=\alpha \phi(s), s=\frac{\beta}{\alpha}$, where $\alpha=\sqrt{a_{i j}(x) y^{i} y^{j}}$ is a Riemannian metric and $\beta=b_{i}(x) y^{i}$ is a 1 -form. The $(\alpha, \beta)$-metrics with $\phi=1+s$ are called Randers metrics. D. Bao and C. Robles have characterized Einstein Randers metrics, and shown that every Einstein Randers metric is necessarily Ricci constant in dimension $n \geq 3$. When $n=3$, a Randers metric is Einstein if and only if it is of constant flag curvature, see [3].

For non-Randers $(\alpha, \beta)$-metrics $F$ with a polynomial function $\phi(s)$ of degree greater than 2 , it was proved that $F$ is an Einstein metric if and only if it is Ricci-flat ([4]). An $(\alpha, \beta)$-metric with $\phi=s^{-1}$ is called a Kropina metric. It was shown that a Kropina metric $F=\frac{\alpha^{2}}{\beta}$ is an Einstein metric if and only if $h$ is an Einstein metric and $W$ a unit Killing form with respect to $h$, where $(h, W)$ is the navigation data of $F([12])$.

The Matsumoto metric is an interesting $(\alpha, \beta)$-metric with $\phi=1 /(1-s)$, introduced by using gradient of slope, speed and gravity in [7]. This metric formulates the model of a Finsler space. Many authors $([1,7,8]$, etc) have studied this metric by different perspectives.

The present paper is devoted to study Einstein Matsumoto metrics, and main results are as follows.

Theorem 1.1. Let $F=\frac{\alpha^{2}}{\alpha-\beta}$ be a non-Riemann Matsumoto metric on an n-dimensional manifold $M$, $n \geq 2$. Then $F$ is an Einstein metric if and only if the followings hold

1) $\alpha$ is an Einstein metric, i.e., $\overline{\text { Ric }}=\lambda \alpha^{2}$,

2) $\beta$ is a conformal form with respect to $\alpha$, i.e., $r_{00}=c \alpha^{2}$,

3)

$$
0=\lambda \alpha^{2}+2 T_{\mid k}^{k}-y^{j} T_{. k \mid j}^{k}+2 T^{j} T_{\cdot j . k}^{k}-T_{. j}^{k} T_{. k}^{j}-\sigma(x) \frac{\alpha^{4}}{(\alpha-\beta)^{2}},
$$

where $\overline{\text { Ric }}$ denotes the Ricci curvature of $\alpha, \lambda=\lambda(x), c=c(x)$ are functions on $M$,

$$
T^{i}=-\frac{\alpha^{2}}{2 \beta-\alpha} s_{0}^{i}-\frac{3 \alpha^{3}}{3 \beta-\left(2 b^{2}+1\right) \alpha}\left(\frac{2}{2 \beta-\alpha} s_{0}+c\right) b^{i}+\frac{\alpha(4 \beta-\alpha)}{2\left(3 \beta-\left(2 b^{2}+1\right) \alpha\right)}\left(\frac{2}{2 \beta-\alpha} s_{0}+c\right) y^{i},
$$

and"|" and"." denote the horizontal covariant derivative and vertical covariant derivative with respect to $\alpha$, respectively.

Supported partially by NNSFC(No. 11171297). 
Notations here can be referred to (2.4) and (2.5) below.

Remark. M. Rafie-Rad, etc., also discussed Einstein Matsumoto metrics. Unfortunately, the computation and results in [10] are wrong because they neglected $b^{2}$ in $a_{i}(i=0, \ldots, 14)$. Theorem 1.1 is the corrected version of Theorem 1 in [10].

Theorem 1.2. Let $F=\frac{\alpha^{2}}{\alpha-\beta}$ be a non-Riemannian Matsumoto metric on an n-dimensional manifold $M, n \geq 3$. Suppose the length of $\beta$ with respect to $\alpha$ is constant. Then $F$ is an Einstein metric if and only if $\alpha$ is Ricci-flat and $\beta$ is parallel with respect to $\alpha$. In this case, $F$ is Ricci-flat.

Theorem 1.3. Let $F=\frac{\alpha^{2}}{\alpha-\beta}$ be a non-Riemannian Matsumoto metric on an $n$-dimensional manifold $M, n \geq 3$. Suppose $\beta^{\sharp}$, which is dual to $\beta$, is a homothetic vector field, i.e., $r_{00}=c \alpha^{2}$, where $c=$ constant. Then $F$ is an Einstein metric if and only if $\alpha$ is Ricci-flat and $\beta$ is parallel with respect to $\alpha$. In this case, $F$ is Ricci-flat.

For an $(\alpha, \beta)$-metric, the form $\beta$ is said to be Killing (resp. closed) form if $r_{i j}=0$ (resp. $s_{i j}=0$ ). $\beta$ is said to be a constant Killing form if it is a Killing form and has constant length with respect to $\alpha$, equivalently $r_{i j}=0$ and $s_{i}=0$.

Remark. B. Rezaei, etc., discussed Einstein Matsumoto metrics with constant Killing form in [11. Meanwhile, they got wrong results. Theorem 1.2 and Theorem 1.3 generalize their study.

For the S-curvature with respect to the Busemann-Hausdorff volume form ([5]), we have following

Theorem 1.4. Let $F=\frac{\alpha^{2}}{\alpha-\beta}$ be a non-Riemannian Matsumoto metric on an n-dimensional manifold $M, n \geq 2$. Then $S$-curvature vanishes if and only if $\beta$ is a constant Killing form.

From above theorems, we can easily get the following

Corollary 1.1. Let $F=\frac{\alpha^{2}}{\alpha-\beta}$ be a non-Riemannian Matsumoto metric on an n-dimensional manifold $M, n \geq 3$. Suppose $F$ is an Einstein metric. Then $S$-curvature vanishes if and only if $\alpha$ is Ricci-flat and $\beta$ is parallel with respect to $\alpha$. In this case, $F$ is Ricci-flat.

The content of this paper is arranged as follows. In $\$ 2$ we introduce essential curvatures of Finsler metrics, as well as notations and conventions. And we give the spray coefficients of Matsumoto metrics. Theorem 1.1 is proved in 93 . In $\$$, we first give the necessary and sufficient conditions for Matsumoto metrics to be Einstein under the hypothesis condition that $\beta$ is a constant Killing form. By using it, Theorem 1.2 and Theorem 1.3 are proved. A nontrivial example of Ricci flat Matsumoto metrics is shown. By the way, we characterize Matsumoto metrics $F$ with constant Killing form $\beta$, which are of constant flag curvature. In $\$ 5$ we investigate the $S$-curvature of Matsumoto metrics and Theorem 1.4 is proved. In the last Section 96 we list the coefficients appeared in the proof of Theorem 1.1 .

\section{Preliminaries}

Let $F$ be a Finsler metric on an $n$-dimensional manifold $M$ and $G^{i}$ the geodesic coefficients of $F$, which are defined by

$$
G^{i}:=\frac{1}{4} g^{i l}\left\{\left[F^{2}\right]_{x^{k} y^{l}} y^{k}-\left[F^{2}\right]_{x^{l}}\right\} .
$$

For any $x \in M$ and $y \in T_{x} M \backslash\{0\}$, the Riemann curvature $\mathbf{R}_{y}:=R_{k}^{i} \frac{\partial}{\partial x^{i}} \otimes d x^{k}$ is defined by

$$
R_{k}^{i}:=2 \frac{\partial G^{i}}{\partial x^{k}}-\frac{\partial^{2} G^{i}}{\partial x^{j} \partial y^{k}} y^{j}+2 G^{j} \frac{\partial^{2} G^{i}}{\partial y^{j} \partial y^{k}}-\frac{\partial G^{i}}{\partial y^{j}} \frac{\partial G^{j}}{\partial y^{k}} .
$$

Ricci curvature is the trace of the Riemann curvature, which is defined by

$$
\text { Ric }:=R_{k}^{k} \text {. }
$$

A Finsler metric $F$ is called an Einstein metric with Einstein scalar $\sigma$ if

$$
\text { Ric }=\sigma F^{2}
$$


where $\sigma=\sigma(x)$ is a scalar function on $M$. In particular, $F$ is said to be Ricci constant (resp. Ricci flat) if $F$ satisfies (2.3) where $\sigma=$ const. (resp. $\sigma=0$ ).

By definition, an $(\alpha, \beta)$-metric on $M$ is expressed in the form $F=\alpha \phi(s), s=\frac{\beta}{\alpha}$, where $\alpha=$ $\sqrt{a_{i j}(x) y^{i} y^{j}}$ is a positive definite Riemannian metric and $\beta=b_{i}(x) y^{i}$ is a 1 -form. It is known that $(\alpha, \beta)$-metric with $\left\|\beta_{x}\right\|_{\alpha}<b_{0}$ is a Finsler metric if and only if $\phi=\phi(s)$ is a positive smooth function on an open interval $\left(-b_{0}, b_{0}\right)$ satisfying the following condition (see [5])

$$
\phi(s)-s \phi^{\prime}(s)+\left(b^{2}-s^{2}\right) \phi^{\prime \prime}(s)>0, \forall|s| \leq b<b_{0} .
$$

Let

$$
r_{i j}=\frac{1}{2}\left(b_{i \mid j}+b_{j \mid i}\right), s_{i j}=\frac{1}{2}\left(b_{i \mid j}-b_{j \mid i}\right),
$$

where "|" denotes the horizontal covariant derivative with respect to $\alpha .{ }^{[5]}$ Denote

$$
\begin{aligned}
r_{j}^{i} & :=a^{i k} r_{k j}, r_{j}:=b^{i} r_{i j}, r:=r_{i j} b^{i} b^{j}=b^{j} r_{j}, r^{i}:=a^{i j} r_{j} \\
s_{j}^{i} & :=a^{i k} s_{k j}, s_{j}:=b^{i} s_{i j}, s^{i}:=a^{i j} s_{j}, \\
r_{i 0} & :=r_{i j} y^{j}, r_{00}:=r_{i j} y^{i} y^{j}, r_{0}:=r_{i} y^{i}, s_{i 0}:=s_{i j} y^{j}, s_{0}^{i}:=s_{j}^{i} y^{j}, s_{0}:=s_{i} y^{i},
\end{aligned}
$$

where $\left(a^{i j}\right):=\left(a_{i j}\right)^{-1}$ and $b^{i}:=a^{i j} b_{j}$.

Let $G^{i}$ and $\bar{G}^{i}$ be the geodesic coefficients of $F$ and $\alpha$, respectively. Then we have the following

Lemma $2.1([6])$. For an $(\alpha, \beta)$-metric $F=\alpha \phi(s), s=\frac{\beta}{\alpha}$, the geodesic coefficients $G^{i}$ are given by

$$
G^{i}=\bar{G}^{i}+\alpha Q s_{0}^{i}+\Psi\left(r_{00}-2 \alpha Q s_{0}\right) b^{i}+\frac{1}{\alpha} \Theta\left(r_{00}-2 \alpha Q s_{0}\right) y^{i},
$$

where

$$
\begin{aligned}
Q & :=\frac{\phi^{\prime}}{\phi-s \phi^{\prime}}, \\
\Psi & :=\frac{\phi^{\prime \prime}}{2\left[\phi-s \phi^{\prime}+\left(b^{2}-s^{2}\right) \phi^{\prime \prime}\right]}, \\
\Theta & :=\frac{\phi \phi^{\prime}-s\left(\phi \phi^{\prime \prime}+\phi^{\prime} \phi^{\prime}\right)}{2 \phi\left[\phi-s \phi^{\prime}+\left(b^{2}-s^{2}\right) \phi^{\prime \prime}\right]} .
\end{aligned}
$$

From now on, we consider a special kind of $(\alpha, \beta)$-metrics which is called Matsumoto-metrics with the form

$$
F=\alpha \phi(s), \phi(s):=\frac{1}{1-s}, s=\frac{\beta}{\alpha} .
$$

Let $b_{0}$ be the largest number such that for any $s$ with $|s| \leq b<b_{0}$. From Lemma 3.1 in [6], we have known that $F$ is a Finsler metric if and only if $b=\left\|\beta_{x}\right\|_{\alpha}<b_{0}=\frac{1}{2}$. So we always assume that $\phi$ satisfies this condition.

Now we get the spray coefficients of Matsumoto metrics by using Lemma 2.1.

Proposition 2.1. For the Matsumoto metric $F=\frac{\alpha^{2}}{\alpha-\beta}$, its geodesic coefficients are

$$
\begin{aligned}
G^{i}= & \bar{G}^{i}-\frac{\alpha}{2 s-1} s_{0}^{i}-\frac{1}{3 s-2 b^{2}-1}\left(\frac{2 \alpha}{2 s-1} s_{0}+r_{00}\right) b^{i} \\
& +\frac{4 s-1}{2\left(3 s-2 b^{2}-1\right)}\left(\frac{2 \alpha}{2 s-1} s_{0}+r_{00}\right) \frac{y^{i}}{\alpha} .
\end{aligned}
$$

Proof. For $\phi(s)=\frac{1}{1-s}$ and by a direct computation, we can obtain (2.7) from (2.6). 


\section{Einstein Matsumoto metrics}

By using Proposition 2.1, we now prove Theorem 1.1.

\section{Proof of Theorem 1.1}

Let

$$
G^{i}=\bar{G}^{i}+T^{i}
$$

where

$$
T^{i}=-\frac{\alpha}{2 s-1} s_{0}^{i}-\frac{1}{3 s-2 b^{2}-1}\left(\frac{2 \alpha}{2 s-1} s_{0}+r_{00}\right) b^{i}+\frac{4 s-1}{2\left(3 s-2 b^{2}-1\right)}\left(\frac{2 \alpha}{2 s-1} s_{0}+r_{00}\right) \frac{y^{i}}{\alpha} .
$$

Thus by (2.1), (2.2) and (2.6), the Ricci curvature of $F$ is related to the Ricci curvature of $\alpha$ by

$$
\text { Ric }=\overline{\operatorname{Ric}}+2 T_{\mid k}^{k}-y^{j} T_{. k \mid j}^{k}+2 T^{j} T_{. j . k}^{k}-T_{. j}^{k} T_{. k}^{j},
$$

where $\overline{\text { Ric }}$ denotes the Ricci curvature of $\alpha$," " and "." denote the horizontal covariant derivative and vertical covariant derivative with respect to $\alpha$, respectively. ${ }^{[5]}$

So the necessary and sufficient condition for the Matsumoto metric to be an Einstein metric is

$$
\begin{aligned}
0 & =\operatorname{Ric}-\sigma(x) F^{2} \\
& =\overline{R i c}+2 T_{\mid k}^{k}-y^{j} T_{. k \mid j}^{k}+2 T^{j} T_{. j . k}^{k}-T^{k}{ }_{. j} T_{. k}^{j}-\sigma(x) \frac{\alpha^{2}}{(1-s)^{2}} .
\end{aligned}
$$

Multiplying both sides of (3.2) by $\alpha^{12}(s-1)^{2}(2 s-1)^{4}\left(3 s-2 b^{2}-1\right)^{4}$ and by a quite long computational procedure using Maple program, we obtain

$$
0=\sum_{m=0}^{14} t_{m} \alpha^{m}
$$

where $t_{m}, m=0,1, \ldots, 14$ are as follows

$$
\left\{\begin{aligned}
t_{0} & =144(8 n-11) \beta^{10} r_{00}^{2}, \\
& \cdots \\
t_{14} & =-\left(1+2 b^{2}\right)^{4} s^{j}{ }_{k} s^{k}{ }_{j}-\left(1+2 b^{2}\right)^{4} \sigma-4\left(1+2 b^{2}\right)^{3} s^{k} s_{k} .
\end{aligned}\right.
$$

All the coefficients of $\alpha$ are tedious, listed in Appendix 96 .

If we replace $y$ by $-y$, then $t_{2 m}(-y)=t_{2 m}(y)$ and $t_{2 \bar{m}+1}(-y)=-t_{2 \bar{m}+1}(y)$ for $m=0, \ldots, 7$ and $\bar{m}=0, \ldots, 6$. Hence (3.3) is equivalent to the following

$$
\left\{\begin{array}{l}
0=t_{0}+t_{2} \alpha^{2}+t_{4} \alpha^{4}+t_{6} \alpha^{6}+t_{8} \alpha^{8}+t_{10} \alpha^{10}+t_{12} \alpha^{12}+t_{14} \alpha^{14} \\
0=t_{1}+t_{3} \alpha^{2}+t_{5} \alpha^{4}+t_{7} \alpha^{6}+t_{9} \alpha^{8}+t_{11} \alpha^{10}+t_{13} \alpha^{12}
\end{array}\right.
$$

From the first equation of (3.4), we know that $\alpha^{2}$ divides $t_{0}$. Since $\alpha^{2}$ is an irreducible polynomial in $y$ and $\beta^{10}$ factors into ten linear terms, it must be the case that $\alpha^{2}$ divides $r_{00}^{2}$. Thus $r_{00}=c \alpha^{2}$ for some function $c=c(x)$, i.e., $\beta$ is a conformal form with respect to $\alpha$. So it is easy to get

$$
\left\{\begin{array}{l}
r_{00}=c \alpha^{2}, r_{i j}=c a_{i j}, r_{0 j}=c y_{j}, r_{i}=c b_{i}, r=c b^{2}, r^{i}{ }_{j}=c \delta_{j}^{i}, \\
r_{0 k} s_{0}^{k}=0, r_{0 k} s^{k}=c s_{0}, r_{0}=c \beta, s_{0}^{k} r_{k}=c s_{0}, \\
r_{00 \mid k}=c_{k} \alpha^{2}, r_{00 \mid 0}=c_{0} \alpha^{2}, r_{k}^{k}=n c, r_{0 \mid 0}=c_{0} \beta+c^{2} \alpha^{2},
\end{array}\right.
$$

where $y_{i}:=a_{i j} y^{j}, c_{k}:=\frac{\partial c}{\partial x^{k}}$ and $c_{0}:=c_{k} y^{k}$.

Plugging (3.5) into the first equation of (3.4) and removing the common factor $\alpha^{2}$, we obtain

$$
0=\bar{t}_{0}+\bar{t}_{2} \alpha^{2}+\ldots+\bar{t}_{12} \alpha^{12},
$$

where

$$
\left\{\begin{aligned}
\bar{t}_{0} & =1296 \overline{\operatorname{Ric}} \beta^{10} \\
\bar{t}_{2} & =72\left(225+240 b^{2}+48 b^{4}\right) \beta^{8} \overline{\operatorname{Ric}}+72\left(-151-56 b^{2}+63 n+24 n b^{2}\right) \beta^{9} c_{0} \\
& -72\left(142+56 b^{2}-57 n-24 n b^{2}\right) \beta^{8} s_{0 \mid 0}-144(8 n-21) \beta^{8} s_{0}^{2}-288(8 n-15) \beta^{9} s_{0} c \\
& -1296 \beta^{9} s_{0 \mid k}^{k}-144(-21+8 n) \beta^{10} c^{2} .
\end{aligned}\right.
$$


Due to the irreducibility of $\alpha$, we have $\alpha^{2}$ divides $\overline{R i c}$, i.e., there exists some function $\lambda=\lambda(x)$ such that

$$
\overline{\operatorname{Ric}}=\lambda \alpha^{2} .
$$

It implies that $\alpha$ is an Einstein metric.

Plugging (3.5) and (3.6) into (3.2) yields (1.2).

Conversely, plugging (3.5), (3.6) and (1.2) into (3.1) yields (3.2), which means that $F$ is an Einstein metric. It completes the proof of Theorem 1.1.

Remark. For Riemann curvature and Ricci curvature of $(\alpha, \beta)$-metrics, L. Zhou gave some formulas in [13. However, Cheng has corrected some errors of his formulas in [4]. To avoid making such mistakes, we use the definitions (2.1) and (2.2) of Riemann curvature and Ricci curvatures to compute them.

\section{The Proofs of Theorem 1.2 And Theorem 1.3}

Lemma 4.1. Let $F=\frac{\alpha^{2}}{\alpha-\beta}$ be a non-Riemann Matsumoto metric on an $n$-dimensional manifold $M$, $n \geq 3$. Suppose $\beta$ is a constant Killing form, i.e., $r_{i j}=0, s_{i}=0$. Then $F$ is an Einstein metric if and only if $\alpha$ is Ricci-flat and $\beta$ is parallel with respect to $\alpha$. In this case, $F$ is Ricci-flat.

Proof. If $F$ is an Einstein metric, then (3.3) holds by Theorem 1.1. Removing the common factor $\alpha^{2}(\alpha-2 \beta)\left(3 \beta-2 b^{2} \alpha-\alpha\right)^{4}$ from (3.3), we obtain

$$
\begin{aligned}
0= & -8 \overline{\operatorname{Ric}} \beta^{5}+28 \overline{\operatorname{Ric}} \beta^{4} \alpha+2\left(-19 \overline{\operatorname{Ric}}+4 s^{k}{ }_{0 \mid k} \beta\right) \beta^{3} \alpha^{2}+\left(-24 s^{k}{ }_{0 \mid k} \beta+25 \overline{\operatorname{Ric}}+2 s_{0 k} s^{k}{ }_{0}\right) \beta^{2} \alpha^{3} \\
& +2\left(-4 \overline{\operatorname{Ric}}+13 s^{k}{ }_{0 \mid k} \beta-2 s_{0 k} s^{k}{ }_{0}+s^{j}{ }_{k} s^{k}{ }_{j} \beta^{2}+4 \sigma \beta^{2}\right) \beta \alpha^{4} \\
& +\left(\overline{\operatorname{Ric}}-12 s^{k}{ }_{0 \mid k} \beta+2 s_{0 k} s^{k}{ }_{0}-5 s^{j}{ }_{k} s^{k}{ }_{j} \beta^{2}-12 \sigma \beta^{2}\right) \alpha^{5} \\
& +2\left(2 s^{j}{ }_{k} s^{k}{ }_{j} \beta+s^{k}{ }_{0 \mid k}+3 \sigma \beta\right) \alpha^{6}-\left(s^{j}{ }_{k} s^{k}{ }_{j}+\sigma\right) \alpha^{7} .
\end{aligned}
$$

Obviously, the equation above is equivalent to

(4.1)

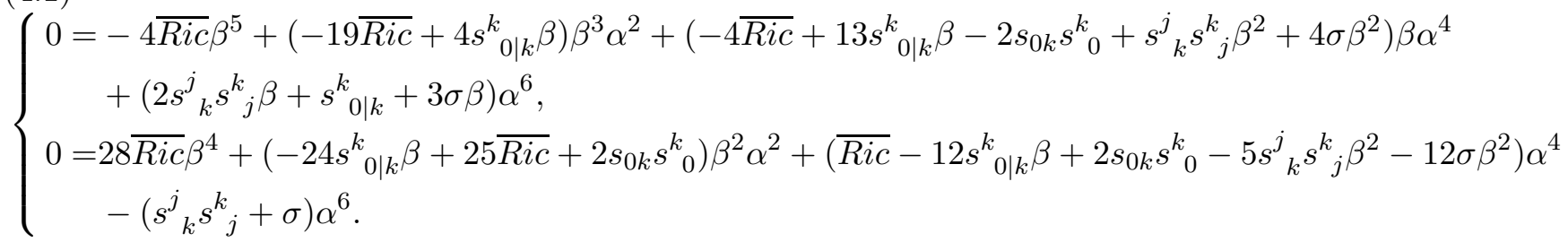

From the first equation of (4.1), we have $\overline{\operatorname{Ric}}=\lambda \alpha^{2}$ for some function $\lambda=\lambda(x)$ on $M$. Using the Bianchi identity, i.e., $b_{j|k| l}-b_{j|l| k}=b^{s} \bar{R}_{j s k l}$, we obtain

$$
s_{k \mid l}^{l}=\lambda b_{k} .
$$

Contracting both sides of (4.2) with $b^{k}$ and $y^{k}$, respectively, we have

$$
\left\{\begin{array}{c}
s^{k}{ }_{j} s^{j}{ }_{k}=-\lambda b^{2}, \\
s^{k}{ }_{0 \mid k}=\lambda \beta .
\end{array}\right.
$$

Substituting (4.3) into (4.1) yields

$$
0=\left(-4 \sigma \beta^{2}+\lambda b^{2} \beta^{2}+6 \lambda \beta^{2}+2 s_{0 k} s_{0}^{k}\right)+\left(-3 \sigma+3 \lambda+2 \lambda b^{2}\right) \alpha^{2},
$$

and

$$
0=2\left(2 \lambda \beta^{2}+s_{0 k} s_{0}^{k}\right) \beta^{2}+\left(-12 \sigma \beta^{2}+2 s_{0 k} s_{0}^{k}+5 \lambda b^{2} \beta^{2}+13 \lambda \beta^{2}\right) \alpha^{2}+\left\{-\sigma+\left(1+b^{2}\right) \lambda\right\} \alpha^{4} .
$$

$3 \times(4.5)-(4.4) \times \alpha^{2}$ yields

$$
0=6\left(2 \lambda \beta^{2}+s_{0 k} s_{0}^{k}\right) \beta^{2}+\left(-32 \sigma \beta^{2}+4 s_{0 k} s_{0}^{k}+14 \lambda b^{2} \beta^{2}+33 \lambda \beta^{2}\right) \alpha^{2}+\lambda b^{2} \alpha^{4} .
$$


Since $\alpha^{2}$ is irreducible polynomial of $y$, we assume that

$$
2 \lambda \beta^{2}+s_{0 k} s_{0}^{k}=h \alpha^{2}
$$

holds for some function $h=h(x)$ on $M$. Differentiating both sides of (4.7) with respect to $y^{i} y^{j}$ yields $4 \lambda b_{i} b_{j}+s_{i k} s^{k}{ }_{j}+s_{j k} s^{k}{ }_{i}=2 h a_{i j}$. Then contracting it with $b^{i} b^{j}$ gives $h=2 \lambda b^{2}$. Thus $s_{0 k} s^{k}{ }_{0}=$ $h \alpha^{2}-2 \lambda \beta^{2}=2 \lambda\left(b^{2} \alpha^{2}-\beta^{2}\right)$. Plugging it into (4.6), we get

$$
0=\left(-32 \sigma+28 \lambda b^{2}+25 \lambda\right) \beta^{2}+9 \lambda b^{2} \alpha^{2} .
$$

Hence (4.8) is equivalent to

$$
\left\{\begin{array}{l}
0=-32 \sigma+28 \lambda b^{2}+25 \lambda \\
0=9 \lambda b^{2}
\end{array}\right.
$$

From the second equation of (4.9), we have $\lambda=0$. Plugging it into the first equation of (4.9) gives $\sigma=0$, i.e., $F$ is Ricci-flat.

Moreover, substituting $\lambda=0$ into (4.3) yields $s_{i j}=0$. Together with $r_{i j}=0$, we have $b_{i \mid j}=0$, i.e., $\beta$ is parallel with respect to $\alpha$.

Converse is obvious. It completes the proof of Lemma 4.1.

It is found that if $\beta$ satisfies $s_{i}=0$ or $r_{00}=c \alpha^{2}$, where $c=$ constant, then $\beta$ is a constant Killing form when $F$ is Einstein. Firstly, we prove the following

Theorem 4.1. Let $F=\frac{\alpha^{2}}{\alpha-\beta}$ be a non-Riemann Matsumoto metric on an n-dimensional manifold $M$, $n \geq 3$. Suppose $\beta$ satisfies $s_{i}=0$. Then $F$ is an Einstein metric if and only if $\alpha$ is Ricci-flat and $\beta$ is parallel with respect to $\alpha$. In this case, $F$ is Ricci-flat.

Proof. If $F$ is an Einstein metric, then $r_{00}=c \alpha^{2}$ and $\overline{R i c}=\lambda \alpha^{2}$ by Theorem 1.1. Plugging $s_{i}=$ $0, r_{00}=c \alpha^{2}, \overline{R i c}=\lambda \alpha^{2}$ into the second equation of (3.4) yields

$$
\begin{aligned}
& 0=432(5-2 n) \beta^{10} c_{0} \\
& +\left\{96\left[48 n-123+(12 n-18) b^{2}\right] c^{2} \beta^{9}-3456\left(2+b^{2}\right) \lambda \beta^{9}-864 b^{k} c_{k} \beta^{9}\right. \\
& \left.\quad-24\left[435 n-602+(354 n-440) b^{2}+(48 n-56) b^{4}\right] c_{0} \beta^{8}\right\} \alpha^{2}+\ldots .
\end{aligned}
$$

From (4.10), we have that $\alpha^{2}$ divides $\beta^{9} c_{0}$. Since $\alpha^{2}$ is irreducible polynomial of $y^{i}$, we have $c_{0}=0$, i.e., $c=$ constant. Plugging it into the first equation of (3.4) yields

$$
\begin{aligned}
0= & 44(21-8 n) \beta^{10} c^{2} \\
& -8 \beta^{6}\left(4832 \beta^{2} c^{2}-81 \beta^{2} s^{j}{ }_{k} s^{k}{ }_{j}-568 n b^{2} \beta^{2} c^{2}+702 \lambda \beta^{2}+128 b^{4} \beta^{2} c^{2}\right. \\
& -1177 n \beta^{2} c^{2}-324 \sigma \beta^{2}-64 n b^{4} \beta^{2} c^{2}+432 \lambda b^{2} \beta^{2}+1376 b^{2} \beta^{2} c^{2} \\
& \left.+270 s_{0 k} s^{k}{ }_{0}+216 b^{2} s_{0 k} s_{0}{ }_{0}\right) \alpha^{2}+\ldots
\end{aligned}
$$

From (4.11), we get $c=0$ for the division reason again. So $\beta$ is a constant Killing form. Thus by Lemma 4.1, we get the necessary conditions.

Sufficiency is obvious. It completes the proof of Theorem 4.1

\section{Proof of Theorem 1.2}

If $F$ is an Einstein metric, then $r_{00}=c \alpha^{2}$ by Theorem 1.1. Thus $r_{k}=c b_{k}$. Since the length of $\beta$, with respect to $\alpha$, is constant, we have $0=b^{2}{ }_{\mid k}=2\left(r_{k}+s_{k}\right)$, i.e., $r_{k}+s_{k}=0$. Hence we get $c b_{k}+s_{k}=0$. Contracting both sides of it with $b^{k}$ yields that $c=0$. Above all, $r_{00}=0$ and $s_{k}=0$, i.e., $\beta$ is a constant Killing form. Thus by Lemma 4.1, we obtain that $\alpha$ is Ricci-flat and $\beta$ is parallel with respect to $\alpha$.

Conversely, if $\alpha$ is Ricci-flat and $\beta$ is parallel with respect to $\alpha$, then the length of $\beta$, with respect to $\alpha$, is constant. Hence by Lemma 4.1, we get $F$ is Einstein. It completes the proof of Theorem 1.2. 
Note that the condition that $s_{k}=0$ in Theorem 4.1 is weaker than one that the length of $\beta$ is constant (with respect to $\alpha$ ) in Theorem 1.2 .

Proof of Theorem 1.3

Assume $F$ is an Einstein metric and $\beta$ is a homothetic form, i.e., $r_{00}=c \alpha^{2}$, where $c=$ constant. Then (3.4) holds, i.e.,

$$
\left\{\begin{array}{l}
0=t_{0}+t_{2} \alpha^{2}+t_{4} \alpha^{4}+t_{6} \alpha^{6}+t_{8} \alpha^{8}+t_{10} \alpha^{10}+t_{12} \alpha^{12}+t_{14} \alpha^{14} \\
0=t_{1}+t_{3} \alpha^{2}+t_{5} \alpha^{4}+t_{7} \alpha^{6}+t_{9} \alpha^{8}+t_{11} \alpha^{10}+t_{13} \alpha^{12}
\end{array}\right.
$$

where

$$
t_{0}=144(8 n-11) c^{2} \beta^{10} \alpha^{4},
$$

and

$$
\begin{aligned}
t_{2} & =12\left\{1085 n-1439+(792 n-1032) b^{2}+64(n-1) b^{4}\right\} c^{2} \beta^{8} \alpha^{4} \\
& +1296 \lambda \beta^{10} \alpha^{2}-288(8 n-14) c^{2} \beta^{10} \alpha^{2}+864 c \beta^{9} s_{0} \alpha^{2} .
\end{aligned}
$$

For division reason again, we have $\alpha^{2}$ can divide $\beta\left(f \beta+g s_{0}\right)$, where $f:=144(8 n-11) c^{2}+1296 \lambda-$ $288(8 n-14) c^{2}, g:=864 c$. So we have $f \beta+g s_{0}=0$. Differentiating both sides of it by $y^{i}$ and contracting it with $b^{i}$ yields $f=0$. So $g=0$ or $s_{0}=0$.

$g=0$ implies that $c=0$. Plugging it into $f=0$ yields $\lambda=0$ and $s^{k}{ }_{0 \mid k}=0$. Substituting all these into (4.12) yields

$$
t_{1}=0, t_{3}=-432(2 n-5) \beta^{9} s_{0 \mid 0},
$$

and

$$
t_{0}=t_{2}=0, t_{4}=72\left\{57 n-142+(24 n-56) b^{2}\right\} \beta^{8} s_{0 \mid 0}-144(8 n-21) \beta^{8} s_{0}^{2} .
$$

From (4.13), we know that $\alpha^{2}$ can divide $s_{0 \mid 0}$. Plugging it into (4.14) yields $\alpha^{2}$ can divide $s_{0}^{2}$. That is $s_{0}^{2}=k(x) \alpha^{2}$, which is a contraction unless $t(x)=0$, i.e., $s_{0}=0$.

Above all, $s_{0}=0$. This is the just case in Theorem 4.1. It completes the proof of Theorem 1.3.

Remark. B. Rezaei, etc., discussed Einstein Matsumoto metrics with constant Killing form. Meanwhile, they got wrong results. Theorem 1.2 and Theorem 1.3 generalize their study and Lemma 4.1 is the corrected version of Theorem 4.2 in [11].

Example. Let $(M, \alpha)$ be an 5-dimensional Riemanian manifold. Consider the Riemannian metric $\alpha=\sqrt{a_{i j}(x) y^{i} y^{j}},(1 \leq i, j \leq 5)$, which, in local coordinate $\left(x^{i}\right)$, can be described as follows

$$
\left(a_{i j}\right)=\left(\begin{array}{ccccc}
\left(x^{4}\right)^{2} & 0 & 0 & 0 & 0 \\
0 & \left(x^{4}\right)^{2} & 0 & 0 & 0 \\
0 & 0 & \left(x^{4}\right)^{-1} & 0 & 0 \\
0 & 0 & 0 & x^{4} & 0 \\
0 & 0 & 0 & 0 & 1
\end{array}\right)
$$

where $x^{4}>0$. A direct computation shows that $\alpha$ is a non-Euclidean Ricci flat metric. And let $\beta=c y^{5}$, where $c$ is a nonzero constant and $c^{2}<\frac{1}{2}$. It is easy to check that such a $\beta$ is parallel with respect to $\alpha$, i.e., $b_{i \mid j}=0$. Define $F=\frac{\alpha^{2}}{\alpha-\beta}$. Thus by Theorem [1.2, we conclude that $F=\frac{\alpha^{2}}{\alpha-\beta}$ is a Ricci-flat Matsumoto metric.

Theorem 4.2. Let $F=\frac{\alpha^{2}}{\alpha-\beta}$ be a non-Riemannian Matsumoto metric on an n-dimensional manifold $M, n \geq 3$. Suppose the length of $\beta$ with respect to $\alpha$ is constant. Then $F$ is of constant flag curvature $K$ if and only if the following conditions hold:

(1) $\alpha$ is a flat metric;

(2) $\beta$ is parallel with respect to $\alpha$.

In this case, $K=0$ and $F$ is locally Minkowskian.

Proof. Suppose that $F$ is of constant flag curvature $K$, i.e.,

$$
R_{k}^{i}=K\left(F^{2} \delta^{i}{ }_{k}-g_{i j} y^{j} y^{k}\right) \text {. }
$$


Then we have

$$
\text { Ric }=\sigma F^{2}, \quad \sigma:=(n-1) K,
$$

which means that $F$ is Einstein. Since the length of $\beta$, with respect to $\alpha$, is constant, by Theorem 1.2, we get $\alpha$ is Ricci-flat and $\beta$ is parallel with respect to $\alpha$. In this case, $F$ is Ricci-flat, which means that $K=0$. So $G^{i}=\bar{G}^{i}$ and $R_{k}^{i}=\bar{R}_{k}^{i}=0$, i.e., $\alpha$ is Euclidean.

Conversely, if $\alpha$ is Euclidean and $\beta$ is parallel with respect to $\alpha$, then $R_{k}^{i}=0$, i.e., $K=0$. It completes the proof of Theorem 4.2 ,

Remark. In literature [9], the proof of Theorem 1 depends on Theorem 3, of which the proof includes the assumption condition that the length of $\beta$ with respect to $\alpha$ is constant, see the step 1 in the proof of Theorem 3 ( $\mathrm{A}$ and $A_{i}(\mathrm{i}=0,1,2, \ldots)$ are some constants) in [9]. So, Theorem 4.2 here is the correct version of Theorem 1 in $[9]$. We do not know what happened to the case that the above assumption is canceled?

\section{5. $S$ CURVATURE}

The $S$-curvature is an important geometric quantity. In this section, we investigate the $S$-curvature of Matsumoto metrics.

For a Finsler metric $F$ and a volume form $d V=\sigma_{F}(x) d x$ on an $n$-dimensional manifold $M$, the $S$-curvature $S$ is given by

$$
S(x, y)=\frac{\partial G^{i}}{\partial y^{i}}-y^{i} \frac{\partial \ln \sigma_{F}}{\partial x^{i}} .
$$

The volume form can be the Busemann-Hausdorff volume form $d V_{B H}=\sigma_{B H} d x$ or the HolmesThompson volume form $d V_{H T}=\sigma_{H T} d x$.

To compute the S-curvature, one should first find a formula for the Busemann-Hausdorff volume forms $d V_{B H}$ and the Holmes-Thompson $d V_{H T}$.

Proposition 5.1. (pro4.1 in [14]) Let $F=\alpha \phi(s), s=\frac{\beta}{\alpha}$, be an $(\alpha, \beta)$-metric on an $n$-dimensional manifold $M$. Denote

$$
f(b):= \begin{cases}\frac{\int_{0}^{\pi} \sin ^{n-2}(t) d t}{\int_{0}^{\pi} \frac{\sin ^{n-2}(t)}{\phi(b \cos t)} d t} & \text { if } d V=d V_{B H}, \\ \frac{\int_{0}^{\pi} \sin ^{n-2}(t) T(b \cos t) d t}{\int_{0}^{\pi} \sin ^{n-2}(t) d t} & \text { if } d V=d V_{H T} .\end{cases}
$$

Then the volume form $d V$ is given by $d V=f(b) d V_{\alpha}$, where $d V_{\alpha}=\sqrt{\operatorname{det}\left(a_{i j}\right)} d x$ denotes the Riemannian volume form of $\alpha, T(s):=\phi\left(\phi-s \phi^{\prime}\right)^{n-2}\left[\phi-s \phi^{\prime}+\left(b^{2}-s^{2}\right) \phi^{\prime \prime}\right]$.

By Proposition 2.1 and Proposition 5.1, we have

$$
\begin{aligned}
\frac{\partial G^{i}}{\partial y^{i}} & =\frac{\partial \bar{G}^{i}}{\partial y^{i}}+\frac{2 s_{0}}{(2 s-1)^{2}}+\frac{6\left(b^{2}-s^{2}\right)}{(2 s-1)\left(3 s-2 b^{2}-1\right)^{2}} s_{0}-\frac{2 s}{(2 s-1)\left(3 s-2 b^{2}-1\right)} s_{0} \\
& +\frac{4\left(b^{2}-s^{2}\right)}{(2 s-1)^{2}\left(3 s-2 b^{2}-1\right)} s_{0}+(n+1) \frac{4 s-1}{(2 s-1)\left(3 s-2 b^{2}-1\right)} s_{0} \\
& +\frac{3\left(b^{2}-s^{2}\right)}{\alpha\left(3 s-2 b^{2}-1\right)^{2}} r_{00}+(n+1) \frac{4 s-1}{2 \alpha\left(3 s-2 b^{2}-1\right)} r_{00}-\frac{2}{\left(3 s-2 b^{2}-1\right)^{2}} r_{0}
\end{aligned}
$$

and

$$
y^{i} \frac{\partial \ln \sigma_{F}}{\partial x^{i}}=y^{i} \frac{\partial \ln \sigma_{\alpha}}{\partial x^{i}}+\Lambda\left(r_{0}+s_{0}\right)
$$

where $\Lambda:=\frac{f^{\prime}(b)}{b f(b)}$. 
Plugging (5.3) and (5.4) into (5.1), we obtain

$$
\begin{aligned}
S & =\frac{2 s_{0}}{(2 s-1)^{2}}+\frac{6\left(b^{2}-s^{2}\right)}{(2 s-1)\left(3 s-2 b^{2}-1\right)^{2}} s_{0}-\frac{2 s}{(2 s-1)\left(3 s-2 b^{2}-1\right)} s_{0} \\
& +\frac{4\left(b^{2}-s^{2}\right)}{(2 s-1)^{2}\left(3 s-2 b^{2}-1\right)} s_{0}+(n+1) \frac{4 s-1}{(2 s-1)\left(3 s-2 b^{2}-1\right)} s_{0} \\
& +\frac{3\left(b^{2}-s^{2}\right)}{\alpha\left(3 s-2 b^{2}-1\right)^{2}} r_{00}+(n+1) \frac{4 s-1}{2 \alpha\left(3 s-2 b^{2}-1\right)} r_{00}-\frac{2}{\left(3 s-2 b^{2}-1\right)^{2}} r_{0} \\
& +\Lambda\left(r_{0}+s_{0}\right) .
\end{aligned}
$$

Proof of Theorem 1.4

Assume that $S=0$. Multiplying both sides of (5.5) by $2 \alpha^{5}(2 s-1)^{2}\left(3 s-2 b^{2}-1\right)^{2}$, we obtain

$$
\begin{aligned}
0= & 24(2 n+1) \beta^{4} r_{00}+\left\{-4\left(13+8 b^{2}+19 n+8 n b^{2}\right) \beta^{3} r_{00}+72 \Lambda \beta^{4} r_{0}+72 \Lambda \beta^{4} s_{0}\right\} \alpha \\
+ & \left\{2\left(19+32 b^{2}+22 n+20 n b^{2}\right) \beta^{2} r_{00}-2\left(60+48 b^{2}\right) \Lambda \beta^{3} r_{0}\right. \\
& \left.-24\left(1-2 n+5 \Lambda+4 \Lambda b^{2}\right) \beta^{3} s_{0}\right\} \alpha^{2} \\
+ & \left\{-\left(11+40 b^{2}+11 n+16 n b^{2}\right) \beta r_{00}+2\left(-8+37 \Lambda+64 \Lambda b^{2}+16 \Lambda b^{4}\right) \beta^{2} r_{0}\right. \\
& \left.+2\left(12-26 n-16 n b^{2}+37 \Lambda+64 \Lambda b^{2}+16 \Lambda b^{4}\right) \beta^{2} s_{0}\right\} \alpha^{3} \\
+ & \left\{\left(1+8 b^{2}+n+2 n b^{2}\right) r_{00}+4\left(4-5 \Lambda-14 \Lambda b^{2}-8 \Lambda b^{4}\right) \beta r_{0}\right. \\
& \left.-2\left(5-8 b^{2}-9 n-12 n b^{2}+10 \Lambda+28 \Lambda b^{2}+16 \Lambda b^{4}\right) \beta s_{0}\right\} \alpha^{4} \\
+ & \left\{-\left(4-2 \Lambda-8 \Lambda b^{2}-8 \Lambda b^{4}\right) r_{0}\right. \\
& \left.+2\left(1-4 b^{2}-n-2 n b^{2}+\Lambda+4 \Lambda b^{2}+4 \Lambda b^{4}\right) s_{0}\right\} \alpha^{5} .
\end{aligned}
$$

(5.6) is equivalent to the following

$$
\left\{\begin{aligned}
0= & 24(2 n+1) \beta^{4} r_{00} \\
+ & \left\{2\left(19+32 b^{2}+22 n+20 n b^{2}\right) \beta^{2} r_{00}-2\left(60+48 b^{2}\right) \Lambda \beta^{3} r_{0}-24\left(1-2 n+5 \Lambda+4 \Lambda b^{2}\right) \beta^{3} s_{0}\right\} \alpha^{2} \\
+ & \left\{\left(1+8 b^{2}+n+2 n b^{2}\right) r_{00}+4\left(4-5 \Lambda-14 \Lambda b^{2}-8 \Lambda b^{4}\right) \beta r_{0}\right. \\
& \left.-2\left(5-8 b^{2}-9 n-12 n b^{2}+10 \Lambda+28 \Lambda b^{2}+16 \Lambda b^{4}\right) \beta s_{0}\right\} \alpha^{4}, \\
0= & \left\{-4\left(13+8 b^{2}+19 n+8 n b^{2}\right) \beta^{3} r_{00}+72 \Lambda \beta^{4} r_{0}+72 \Lambda \beta^{4} s_{0}\right\} \\
+ & \left\{-\left(11+40 b^{2}+11 n+16 n b^{2}\right) \beta r_{00}+2\left(-8+37 \Lambda+64 \Lambda b^{2}+16 \Lambda b^{4}\right) \beta^{2} r_{0}\right. \\
& \left.+2\left(12-26 n-16 n b^{2}+37 \Lambda+64 \Lambda b^{2}+16 \Lambda b^{4}\right) \beta^{2} s_{0}\right\} \alpha^{2} \\
+ & \left\{-\left(4-2 \Lambda-8 \Lambda b^{2}-8 \Lambda b^{4}\right) r_{0}+2\left(1-4 b^{2}-n-2 n b^{2}+\Lambda+4 \Lambda b^{2}+4 \Lambda b^{4}\right) s_{0}\right\} \alpha^{4} .
\end{aligned}\right.
$$

From the first equation of (5.7), we have

$$
r_{00}=c \alpha^{2},
$$

for some function $c=c(x)$ on $M$. So $r_{0}=c \beta$.

Plugging (5.8) and $r_{0}=c \beta$ into (5.7), we obtain

$$
\left\{\begin{aligned}
0= & 24 c\left(1+2 n-5 \Lambda-4 \Lambda b^{2}\right) \beta^{4}-24\left(1-2 n+5 \Lambda+4 \Lambda b^{2}\right) \beta^{3} s_{0} \\
& +\left\{2 c\left(27+32 b^{2}+22 n+20 n b^{2}-10 \Lambda-28 \Lambda b^{2}-16 \Lambda b^{4}\right) \beta^{2}\right. \\
& \left.-2\left(5-8 b^{2}-9 n-12 n b^{2}+10 \Lambda+28 \Lambda b^{2}+16 \Lambda b^{4}\right) \beta s_{0}\right\} \alpha^{2}+c\left(1+8 b^{2}+n+2 n b^{2}\right) \alpha^{4} \\
0= & 72 \Lambda \beta^{4}\left(c \beta+s_{0}\right) .
\end{aligned}\right.
$$

From the second equation of (5.9), we have $c \beta+s_{0}=0$ for $n \geq 2$. Differentiating both sides of it with respect to $y^{i}$ yields $c b_{i}+s_{i}=0$. Contracting it with $b^{i}$ gives $c b^{2}=0$. So $c=0$ and $s_{0}=0$. Thus $r_{00}=0, s_{0}=0$, i.e., $\beta$ is a constant Killing form.

Conversely, if $\beta$ is a constant Killing form, then $S=0$ by (5.5). Thus we have completed the proof of Theorem 1.4.

By Theorem 1.4 and Theorem 1.2, we can directly get Corollary 1.1. 
6. Appendix: Coefficients in (3.3)

$$
\begin{aligned}
& t_{0}=144(8 n-11) \beta^{10} r_{00}^{2} \\
& t_{1}=-96\left\{61 n-82+(20 n-26) b^{2}\right\} \beta^{9} r_{00}^{2}-432(2 n-3) \beta^{10} r_{00 \mid 0} \\
& t_{2}=12\left\{1085 n-1439+(792 n-1032) b^{2}+64(n-1) b^{4}\right\} \beta^{8} r_{00}^{2} \\
& +1296 \beta^{10} \overline{\text { Ric }}-288(8 n-14) \beta^{9} r_{0} r_{00}+864 \beta^{9} s_{0} r_{00}+72\left\{63 n-91+(24 n-32) b^{2}\right\} \beta^{9} r_{00 \mid 0} \text {; } \\
& t_{3}=-864(2 n-1) \beta^{9} r_{0 k} s^{k}{ }_{0}-24\left\{697 n-926+(852 n-1144) b^{2}+(152 n-144) b^{4}\right\} \beta^{7} r_{00}^{2} \\
& -3456\left(2+b^{2}\right) \beta^{9} \overline{R i c}+96\left\{118 n-205+(32 n-44) b^{2}\right\} \beta^{8} r_{00} r_{0}-864 \beta^{9} r_{00} r_{k}^{k} \\
& -48\left\{-16 n+97+16(n-1) b^{2}\right\} \beta^{8} r_{00} s_{0}-864 \beta^{9} b^{k} r_{00 \mid k} \\
& -24\left\{435 n-602+(354 n-440) b^{2}+(48 n-56) b^{4}\right\} \beta^{8} r_{00 \mid 0}+864 \beta^{9} r_{0 \mid 0}-432(2 n-5) \beta^{9} s_{0 \mid 0} ; \\
& \begin{aligned}
t_{4} & =144\left\{57 n-22+(24 n-8) b^{2}\right\} \beta^{8} r_{0 k} s_{0}^{k} \\
& +3\left\{4606 n-6255+(8400 n-12080) b^{2}+(2480 n-2272) b^{4}\right\} \beta^{6} r_{00}^{2} \\
& +216\left(15+4 b^{2}\right)\left(5+4 b^{2}\right) \beta^{8} \overline{R i c}-32\left\{752 n-1301+(440 n-566) b^{2}+32(n-1) b^{4}\right\} \beta^{7} r_{00} r_{0} \\
& +864\left(5+2 b^{2}\right) \beta^{8}\left(r_{00} r^{k}{ }_{k}+b^{k} r_{00 \mid k}\right)-576 \beta^{8} r r_{00} \\
& +8\left\{-413 n+1322+(376 n-664) b^{2}+64(n-1) b^{4}\right\} \beta^{7} r_{00} s_{0} \\
& +4\left\{3473 n-4583+(4512 n-5136) b^{2}+(1320 n-1368) b^{4}+64(n-1) b^{6}\right\} \beta^{7} r_{00 \mid 0} \\
& -864\left(5+2 b^{2}\right) \beta^{8} r_{0 \mid 0}+576 \beta^{8} r_{0}^{2}-1152(2 n-3) \beta^{8} r_{0} s_{0}+72\left\{57 n-142+(24 n-56) b^{2}\right\} \beta^{8} s_{0 \mid 0} \\
& -144(8 n-21) \beta^{8} s_{0}^{2}-1296 \beta^{9} s_{0 \mid k}^{k} ;
\end{aligned} \\
& t_{5}=-24\left\{699 n-178+(636 n-112) b^{2}+(96 n-16) b^{4}\right\} \beta^{7} s^{k}{ }_{0} r_{0 k} \\
& -12\left\{643 n-911+(1642 n-2645) b^{2}+(712 n-656) b^{4}\right\} \beta^{5} r_{00}^{2} \\
& -24\left(917+1560 b^{2}+672 b^{4}+64 b^{6}\right) \beta^{7} \overline{\text { Ric }} \\
& +16\left\{1814 n-3143+(1712 n-2024) b^{2}+(272 n-224) b^{4}\right\} \beta^{6} r_{00} r_{0} \\
& -144\left(65+56 b^{2}+8 b^{4}\right) \beta^{7}\left(b^{k} r_{00 \mid k}+r_{00} r_{k}^{k}\right)+384\left(7+2 b^{2}\right) \beta^{7} r r_{00} \\
& -4\left\{-1487 n+3338+(1240 n-3952) b^{2}+(544 n-736) b^{4}\right\} \beta^{6} r_{00} s_{0} \\
& -\left\{11854 n-14857+(21768 n-22176) b^{2}+(10272 n-9024) b^{4}+(1088 n-896) b^{6}\right\} \beta^{6} r_{00 \mid 0} \\
& +144\left(65+56 b^{2}+8 b^{4}\right) \beta^{7} r_{0 \mid 0}-384\left(7+2 b^{2}\right) \beta^{7} r_{0}^{2}+48\left\{212 n-321+(64 n-72) b^{2}\right\} \beta^{7} r_{0} s_{0} \\
& -12\left\{699 n-1738+(636 n-1456) b^{2}+(96 n-208) b^{4}\right\} \beta^{7} s_{0 \mid 0}-324 \beta^{7} s_{0 k} s^{k}{ }_{0} \\
& +864 \beta^{8}\left(r_{k} s_{0}^{k}+r_{0 k} s^{k}-b^{k} s_{0 \mid k}-r_{k}^{k} s_{0}\right)+216\left(29+16 b^{2}\right) \beta^{8} s^{k}{ }_{0 \mid k}-432(2 n-3) \beta^{8} s^{k}{ }_{0} s_{k} \\
& +48\left\{114 n-239+(24 n-52) b^{2}\right\} \beta^{7} s_{0}^{2} \text {; }
\end{aligned}
$$




$$
\begin{aligned}
t_{6} & =4\left\{4849 n-516+(7116 n+96) b^{2}+(2352 n+96) b^{4}+128 n b^{6}\right\} \beta^{6} s_{0}^{k} r_{0 k} \\
& +3 / 4\left\{3965 n-5929+(13592 n-25576) b^{2}+(8096 n-7936) b^{4}\right\} \beta^{4} r_{00}^{2} \\
& +\left(19225+46208 b^{2}+32064 b^{4}+6656 b^{6}+256 b^{8}\right) \beta^{6} \overline{R i c} \\
& -12\left\{1828 n-3201+(2464 n-2616) b^{2}+(640 n-384) b^{4}\right\} \beta^{5} r_{00} r_{0} \\
& +32\left(361+501 b^{2}+156 b^{4}+8 b^{6}\right) \beta^{6}\left(r^{k}{ }_{k} r_{00}+b^{k} r_{00 \mid k}\right)-32\left(167+104 b^{2}+8 b^{4}\right) \beta^{6} r_{00} r \\
& +6\left\{-973 n+1745+(776 n-4336) b^{2}+(656 n-1216) b^{4}\right\} \beta^{5} r_{00} s_{0} \\
& +3 / 2\left\{4525 n-5373+(10968 n-9696) b^{2}+(7392 n-5088) b^{4}+(1280 n-768) b^{6}\right\} \beta^{5} r_{00 \mid 0} \\
& -32\left(361+501 b^{2}+156 b^{4}+8 b^{6}\right) \beta^{6} r_{0 \mid 0}+32\left(167+104 b^{2}+8 b^{4}\right) \beta^{6} r_{0}^{2} \\
& -8\left\{2372 n-3657+(1568 n-1656) b^{2}+(128 n-96) b^{4}\right\} \beta^{6} s_{0} r_{0}+432\left(9+4 b^{2}\right) \beta^{7} s_{0} r^{k}{ }_{k} \\
& +2\left\{4849 n-12068+(7116 n-15936) b^{2}+(2352 n-4896) b^{4}+(128 n-256) b^{6}\right\} \beta^{6} s_{0 \mid 0} \\
& +432\left(9+4 b^{2}\right) \beta^{7}\left(b^{k} s_{0 \mid k}-r_{0 k} s^{k}-s^{k}{ }_{0} r_{k}\right)-108\left(121+144 b^{2}+32 b^{4}\right) \beta^{7} s^{k}{ }_{0 \mid k}-576 \beta^{7} s_{0} r \\
& +54\left(23+16 b^{2}\right) \beta^{6} s^{k}{ }_{0} s_{0 k}+72\left\{51 n-73+(24 n-32) b^{2}\right\} \beta^{7} s^{k}{ }_{0} s_{k} \\
& -4\left\{2737 n-4424+(1240 n-1208) b^{2}+(64 n-128) b^{4}\right\} \beta^{6} s_{0}^{2}-324 \beta^{8} s^{j}{ }_{k} s^{k}{ }_{j}-1296 \beta^{8} \sigma ;
\end{aligned}
$$

$$
\begin{aligned}
t_{7} & =-2\left(401+3504 b^{2}+2400 b^{4}+256 b^{6}+7005 n+14652 n b^{2}+7920 n b^{4}+960 n b^{6}\right) \beta^{5} s^{k}{ }_{0} r_{0 k} \\
& -3\left(-411-2650 b^{2}-1016 b^{4}+263 n+1170 n b^{2}+916 n b^{4}\right) \beta^{3} r_{00}^{2} \\
& -2\left(5651+17932 b^{2}+17760 b^{4}+6016 b^{6}+512 b^{8}\right) \beta^{5} \overline{R i c} \\
& +6\left(-3225-2988 b^{2}-384 b^{4}+1798 n+3232 n b^{2}+1216 n b^{4}\right) \beta^{4} r_{00} r_{0} \\
& -2\left(4483+8880 b^{2}+4512 b^{4}+512 b^{6}\right) \beta^{5}\left(r_{00} r^{k}{ }_{k}+b^{k} r_{00 \mid k}\right)+32\left(37+8 b^{2}\right)\left(5+4 b^{2}\right) \beta^{5} r_{00} r \\
& -3\left(1817-8560 b^{2}-3328 b^{4}-1135 n+968 n b^{2}+1328 n b^{4}\right) \beta^{4} r_{00} s_{0} \\
& -3 / 2\left(-1983-3960 b^{2}-2232 b^{4}-384 b^{6}+1763 n+5394 n b^{2}+4848 n b^{4}+1216 n b^{6}\right) \beta^{4} r_{00 \mid 0} \\
& +2\left(4483+8880 b^{2}+4512 b^{4}+512 b^{6}\right) \beta^{5} r_{0 \mid 0}-32\left(37+8 b^{2}\right)\left(5+4 b^{2}\right) \beta^{5} r_{0}^{2} \\
& -4\left(-7769-5144 b^{2}-560 b^{4}+4884 n+5280 n b^{2}+960 n b^{4}\right) \beta^{5} s_{0} r_{0} \\
& -\left(-17531-32016 b^{2}-15648 b^{4}-1792 b^{6}+7005 n+14652 n b^{2}+7920 n b^{4}+960 n b^{6}\right) \beta^{5} s_{0 \mid 0} \\
& +72\left(103+100 b^{2}+16 b^{4}\right) \beta^{6}\left(s^{k} r_{0 k}+s^{k}{ }_{0} r_{k}-s_{0} r^{k}{ }_{k}-b^{k} s_{0 \mid k}\right) \\
& +6\left(2579+4944 b^{2}+2400 b^{4}+256 b^{6}\right) \beta^{6} s^{k}{ }_{0 \mid k}-216\left(9+14 b^{2}+4 b^{4}\right) \beta^{5} s^{k}{ }_{0} s_{0 k} \\
& -12\left(-739-688 b^{2}-112 b^{4}+546 n+564 n b^{2}+96 n b^{4}\right) \beta^{6} s^{k}{ }_{0} s_{k} \\
& +4\left(-3622+656 b^{2}+320 b^{4}+3003 n+2184 n b^{2}+240 n b^{4}\right) \beta^{5} s_{0}^{2} \\
& +8\left(-7769-5144 b^{2}-560 b^{4}+4884 n+5280 n b^{2}+960 n b^{4}\right) \beta^{5} s_{0} r_{0}+96\left(25+8 b^{2}\right) \beta^{6} s_{0} r \\
& +108\left(13+8 b^{2}\right) \beta^{7} s^{j}{ }_{k} s^{k}{ }_{j}+864\left(5+4 b^{2}\right) \beta^{7} \sigma+432 \beta^{7} s^{k} s_{k} ;
\end{aligned}
$$




$$
\begin{aligned}
t_{8} & =2\left(769+4596 b^{2}+4512 b^{4}+896 b^{6}+3285 n+9126 n b^{2}+7128 n b^{4}+1440 n b^{6}\right) \beta^{4} r_{0 k} s^{k}{ }_{0} \\
& +3 / 2\left(-145-1436 b^{2}-684 b^{4}+93 n+520 n b^{2}+518 n b^{4}\right) \beta^{2} r_{00}^{2} \\
& +\left(4535+18184 b^{2}+24024 b^{4}+11776 b^{6}+1664 b^{8}\right) \beta^{4} \overline{R i c} \\
& -12\left(-539-509 b^{2}+16 b^{4}+288 n+660 n b^{2}+336 n b^{4}\right) \beta^{3} r_{00} r_{0} \\
& +2\left(2273+6006 b^{2}+4416 b^{4}+832 b^{6}\right) \beta^{4}\left(r_{00} r^{k}{ }_{k}+b^{k} r_{00 \mid k}\right)-4\left(1001+1472 b^{2}+416 b^{4}\right) \beta^{4} r_{00} r \\
& +12\left(165-1293 b^{2}-672 b^{4}-100 n+110 n b^{2}+206 n b^{4}\right) \beta^{3} r_{00} s_{0} \\
& +3\left(-247-492 b^{2}-186 b^{4}+16 b^{6}+231 n+864 n b^{2}+990 n b^{4}+336 n b^{6}\right) \beta^{3} r_{00 \mid 0} \\
& -2\left(2273+6006 b^{2}+4416 b^{4}+832 b^{6}\right) \beta^{4} r_{0 \mid 0}+4\left(1001+1472 b^{2}+416 b^{4}\right) \beta^{4} r_{0}^{2} \\
& -2\left(-10175-8360 b^{2}-1040 b^{4}+6084 n+9504 n b^{2}+2880 n b^{4}\right) \beta^{4} r_{0} s_{0} \\
& +4\left(1961+3108 b^{2}+1104 b^{4}+64 b^{6}\right) \beta^{5}\left(r^{k}{ }_{k} s_{0}+b^{k} s_{0 \mid k}-r_{0 k} s^{k}-s^{k}{ }_{0} r_{k}\right) \\
& +\left(-8323-19428 b^{2}-13152 b^{4}-2432 b^{6}+3285 n+9126 n b^{2}+7128 n b^{4}+1440 n b^{6}\right) \beta^{4} s_{0 \mid 0} \\
& -16\left(718+1961 b^{2}+1554 b^{4}+368 b^{6}+16 b^{8}\right) \beta^{5} s^{k}{ }_{0 \mid k}+6\left(269+696 b^{2}+456 b^{4}+64 b^{6}\right) \beta^{4} s_{0}^{k} s_{0 k} \\
& +2\left(-4075-5856 b^{2}-2064 b^{4}-128 b^{6}+3211 n+5424 n b^{2}+2064 n b^{4}+128 n b^{6}\right) \beta^{5} s^{k}{ }_{0} s_{k} \\
& -\left(-7009+13792 b^{2}+7744 b^{4}+512 b^{6}+7881 n+8088 n b^{2}+1392 n b^{4}\right) \beta^{4} s_{0}^{2} \\
& -27\left(95+128 b^{2}+32 b^{4}\right) \beta^{6} s^{j}{ }_{k} s^{k}{ }_{j}-216\left(29+48 b^{2}+16 b^{4}\right) \beta^{6} \sigma-864\left(2+b^{2}\right) \beta^{6} s^{k} s_{k} \\
& -16\left(259+184 b^{2}+16 b^{4}\right) \beta^{5} r s_{0} ;
\end{aligned}
$$

$$
\begin{aligned}
t_{9} & =-4\left(211+1428 b^{2}+1938 b^{4}+608 b^{6}+501 n+1764 n b^{2}+1854 n b^{4}+552 n b^{6}\right) \beta^{3} s_{0}^{k} r_{0 k} \\
& -3\left(-7-114 b^{2}-68 b^{4}+5 n+34 n b^{2}+42 n b^{4}\right) \beta r_{00}^{2} \\
& -4\left(1+2 b^{2}\right)\left(307+894 b^{2}+756 b^{4}+176 b^{6}\right) \beta^{3} \frac{\overline{R i c}}{} \\
& +12\left(-116-99 b^{2}+56 b^{4}+58 n+164 n b^{2}+108 n b^{4}\right) \beta^{2} r_{00} r_{0} \\
& -4\left(377+1272 b^{2}+1266 b^{4}+352 b^{6}\right) \beta^{3}\left(r_{00} r^{k}{ }_{k}+b^{k} r_{00 \mid k}-r_{0 \mid 0}\right) \\
& +16\left(106+211 b^{2}+88 b^{4}\right) \beta^{3} r r_{00}-6\left(85-934 b^{2}-636 b^{4}-41 n+72 n b^{2}+158 n b^{4}\right) \beta^{2} r_{00} s_{0} \\
& -3\left(-40-68 b^{2}+34 b^{4}+56 b^{6}+39 n+174 n b^{2}+246 n b^{4}+108 n b^{6}\right) \beta^{2} r_{00 \mid 0} \\
& -16\left(106+211 b^{2}+88 b^{4}\right) \beta^{3} r_{0}^{2}+16\left(-530-479 b^{2}-17 b^{4}+294 n+618 n b^{2}+276 n b^{4}\right) \beta^{3} s_{0} r_{0} \\
& +8\left(481+568 b^{2}+112 b^{4}\right) \beta^{4} r s_{0} \\
& -2\left(-1297-3660 b^{2}-3126 b^{4}-800 b^{6}+501 n+1764 n b^{2}+1854 n b^{4}+552 n b^{6}\right) \beta^{3} s_{0 \mid 0} \\
& +4\left(1261+2886 b^{2}+1704 b^{4}+224 b^{6}\right) \beta^{4}\left(s^{k} r_{0 k}+s^{k}{ }_{0} r_{k}-r^{k}{ }_{k} s_{0}-b^{k} s_{0 \mid k}\right) \\
& +2\left(2779+10088 b^{2}+11544 b^{4}+4544 b^{6}+448 b^{8}\right) \beta^{4} s^{k}{ }_{0 \mid k} \\
& -4\left(1+2 b^{2}\right)\left(193+342 b^{2}+132 b^{4}+8 b^{6}\right) \beta^{3} s^{k}{ }_{0} s_{0 k} \\
& -2\left(-2245-4200 b^{2}-2280 b^{4}-320 b^{6}+1897 n+4614 n b^{2}+2928 n b^{4}+416 n b^{6}\right) \beta^{4} s^{k}{ }_{0} s_{k} \\
& +4\left(-549+3536 b^{2}+2828 b^{4}+368 b^{6}+789 n+1050 n b^{2}+240 n b^{4}\right) \beta^{3} s_{0}^{2} \\
& +6\left(431+948 b^{2}+528 b^{4}+64 b^{6}\right) \beta^{5} s^{j}{ }_{k} s^{k}{ }_{j}+24\left(5+4 b^{2}\right)\left(43+76 b^{2}+16 b^{4}\right) \beta^{5} \sigma \\
& +36\left(79+88 b^{2}+16 b^{4}\right) \beta^{5} s^{k} s_{k} ;
\end{aligned}
$$




$$
\begin{aligned}
t_{10} & =4\left(59+480 b^{2}+870 b^{4}+400 b^{6}+96 n+414 n b^{2}+558 n b^{4}+228 n b^{6}\right) \beta^{2} r_{0 k} s_{0}^{k} \\
& +3 / 4\left\{n-1+(8 n-32) b^{2}+(12 n-24) b^{4}\right\} r_{00}^{2}+\left(215+404 b^{2}+164 b^{4}\right)\left(1+2 b^{2}\right)^{2} \beta^{2} \overline{R i c} \\
& -4\left(-44-29 b^{2}+64 b^{4}+20 n+68 n b^{2}+56 n b^{4}\right) \beta r_{00} r_{0} \\
& -8\left(55+142 b^{2}+82 b^{4}\right) \beta^{2} r_{00} r+2\left(43-554 b^{2}-488 b^{4}-13 n+44 n b^{2}+104 n b^{4}\right) \beta r_{00} s_{0} \\
& +4\left(1+2 b^{2}\right)\left(79+172 b^{2}+82 b^{4}\right) \beta^{2}\left(b^{k} r_{00 \mid k}+r_{00} r_{k}^{k}\right) \\
& +1 / 2\left(1+2 b^{2}\right)\left(-23+22 b^{2}+64 b^{4}+23 n+74 n b^{2}+56 n b^{4}\right) \beta r_{00 \mid 0} \\
& -4\left(1+2 b^{2}\right)\left(79+172 b^{2}+82 b^{4}\right) \beta^{2} r_{0 \mid 0}+8\left(55+142 b^{2}+82 b^{4}\right) \beta^{2} r_{0}^{2} \\
& -4\left(-553-496 b^{2}+158 b^{4}+276 n+744 n b^{2}+456 n b^{4}\right) \beta^{2} s_{0} r_{0} \\
& +8\left(253+780 b^{2}+678 b^{4}+152 b^{6}\right) \beta^{3}\left(s_{0} r^{k}{ }_{k}+b^{k} s_{0 \mid k}-r_{0 k} s^{k}-s^{k}{ }_{0} r_{k}\right) \\
& +2\left(-257-840 b^{2}-834 b^{4}-256 b^{6}+96 n+414 n b^{2}+558 n b^{4}+228 n b^{6}\right) \beta^{2} s_{0 \mid 0} \\
& -4\left(1+2 b^{2}\right)\left(439+1146 b^{2}+828 b^{4}+152 b^{6}\right) \beta^{3} s^{k}{ }_{0 \mid k}-32\left(65+113 b^{2}+38 b^{4}\right) \beta^{3} s_{0} r \\
& +2\left(107+116 b^{2}+20 b^{4}\right)\left(1+2 b^{2}\right)^{2} \beta^{2} s^{k}{ }_{0} s_{0 k}-4\left(625+1158 b^{2}+480 b^{4}+32 b^{6}\right) \beta^{4} s^{k} s_{k} \\
& +4\left(-383-804 b^{2}-510 b^{4}-112 b^{6}+347 n+1128 n b^{2}+1050 n b^{4}+256 n b^{6}\right) \beta^{3} s_{k} s^{k}{ }_{0} \\
& -2\left(-255+3368 b^{2}+3668 b^{4}+752 b^{6}+375 n+600 n b^{2}+150 n b^{4}\right) \beta^{2} s_{0}^{2} \\
& -\left(1579+5000 b^{2}+4632 b^{4}+1280 b^{6}+64 b^{8}\right) \beta^{4} s^{j}{ }_{k} s^{k}{ }_{j} \\
& -\left(2641+9344 b^{2}+10176 b^{4}+3584 b^{6}+256 b^{8}\right) \beta^{4} \sigma ;
\end{aligned}
$$

$$
\begin{aligned}
t_{11} & =-2\left(1+2 b^{2}\right)\left(17+134 b^{2}+128 b^{4}+21 n+66 n b^{2}+48 n b^{4}\right) \beta s_{0}^{k} r_{0 k} \\
& +2\left(1+2 b^{2}\right)\left(-5+8 b^{2}+2 n+4 n b^{2}\right) r_{00} r_{0}-2\left(19+20 b^{2}\right)\left(1+2 b^{2}\right)^{2} \beta\left(r_{00} r_{k}^{k}+b^{k} r_{00 \mid k}\right) \\
& +16\left(4+5 b^{2}\right)\left(1+2 b^{2}\right) \beta r r_{00}-\left(7-92 b^{2}-104 b^{4}-n+8 n b^{2}+20 n b^{4}\right) r_{00} s_{0} \\
& -2\left(11+10 b^{2}\right)\left(1+2 b^{2}\right)^{3} \beta \overline{R i c}-1 / 2\left(1+2 b^{2}\right)^{2}\left(-1+4 b^{2}+n+2 n b^{2}\right) r_{00 \mid 0} \\
& +2\left(19+20 b^{2}\right)\left(1+2 b^{2}\right)^{2} \beta r_{0 \mid 0}-16\left(4+5 b^{2}\right)\left(1+2 b^{2}\right) \beta r_{0}^{2} \\
& +4\left(-83-68 b^{2}+88 b^{4}+36 n+120 n b^{2}+96 n b^{4}\right) \beta r_{0} s_{0}+16\left(41+98 b^{2}+50 b^{4}\right) \beta^{2} r s_{0} \\
& -\left(1+2 b^{2}\right)\left(-59-98 b^{2}-32 b^{4}+21 n+66 n b^{2}+48 n b^{4}\right) \beta s_{0 \mid 0} \\
& +16\left(1+2 b^{2}\right)\left(31+61 b^{2}+25 b^{4}\right) \beta^{2}\left(r_{0 k} s^{k}+s^{k}{ }_{0} r_{k}-r^{k}{ }_{k} s_{0}-b^{k} s_{0 \mid k}\right) \\
& +2\left(175+292 b^{2}+100 b^{4}\right)\left(2 b^{2}+1\right)^{2} \beta^{2} s^{k}{ }_{0 \mid k}-16\left(2+b^{2}\right)\left(1+2 b^{2}\right)^{3} \beta s^{k}{ }_{0} s_{0 k} \\
& -4\left(-80-156 b^{2}-42 b^{4}+8 b^{6}+77 n+318 n b^{2}+402 n b^{4}+148 n b^{6}\right) \beta^{2} s_{k} s^{k}{ }_{0} \\
& +4\left(-23+392 b^{2}+560 b^{4}+160 b^{6}+24 n+42 n b^{2}+6 n b^{4}\right) \beta s_{0}^{2} \\
& +24\left(1+2 b^{2}\right)\left(25+56 b^{2}+32 b^{4}+4 b^{6}\right) \beta^{3} s^{j}{ }_{k} s^{k}{ }_{j}+4\left(5+4 b^{2}\right)\left(1+2 b^{2}\right)\left(43+76 b^{2}+16 b^{4}\right) \beta^{3} \sigma \\
& +24\left(53+144 b^{2}+102 b^{4}+16 b^{6}\right) \beta^{3} s^{k} s_{k} ;
\end{aligned}
$$

$$
\begin{aligned}
t_{12} & =2\left(1+2 b^{2}\right)^{2}\left(1+8 b^{2}+n+2 n b^{2}\right) r_{0 k} s_{0}^{k}+\left(1+2 b^{2}\right)^{4} \overline{R i c}+2\left(1+2 b^{2}\right)^{3}\left(r_{00} r_{k}^{k}+b^{k} r_{00 \mid k}\right) \\
& -4\left(1+2 b^{2}\right)^{2} r r_{00}-2\left(1+2 b^{2}\right)^{3} r_{0 \mid 0}+4\left(1+2 b^{2}\right)^{2} r_{0}^{2}-2\left(1+2 b^{2}\right)\left\{4 n-11+(8 n+14) b^{2}\right\} r_{0} s_{0} \\
& +4\left(1+2 b^{2}\right)^{2}\left(17+16 b^{2}\right) \beta\left(s_{0} r^{k}{ }_{k}+b^{k} s_{0 \mid k}-s^{k} r_{0 k}-s^{k}{ }_{0} r_{k}\right)-8\left(5+4 b^{2}\right)\left(1+2 b^{2}\right)^{3} \beta s^{k}{ }_{0 \mid k} \\
& -16\left(1+2 b^{2}\right)\left(7+8 b^{2}\right) \beta s_{0} r+\left(1+2 b^{2}\right)^{2}\left(-3+n+2 n b^{2}\right) s_{0 \mid 0} \\
& +2\left(1+2 b^{2}\right)^{4} s^{k}{ }_{0} s_{0 k}+2\left(1+2 b^{2}\right)\left\{19 n-19+(58 n+14) b^{2}+(40 n+32) b^{4}\right\} \beta s^{k}{ }_{0} s_{k} \\
& +\left\{-5 n+9-(8 n+144) b^{2}+(4 n-264) b^{4}-96 b^{6}\right\} s_{0}^{2}-8\left(1+2 b^{2}\right)\left(47+80 b^{2}+26 b^{4}\right) \beta^{2} s^{k} s_{k} \\
& -\left(139+196 b^{2}+52 b^{4}\right)\left(1+2 b^{2}\right)^{2} \beta^{2} s^{j}{ }_{k} s^{k}{ }_{j}-6\left(29+48 b^{2}+16 b^{4}\right)\left(1+2 b^{2}\right)^{2} \beta^{2} \sigma
\end{aligned}
$$




$$
\begin{aligned}
t_{13} & =8\left(1+2 b^{2}\right)^{2} r s_{0}+4\left(1+2 b^{2}\right)^{3}\left(s^{k} r_{0 k}+s_{0}^{k} r_{k}-s_{0} r_{k}^{k}-b^{k} s_{0 \mid k}\right)+2\left(1+2 b^{2}\right)^{4} s_{0 \mid k}^{k} \\
& -2\left(1+2 b^{2}\right)^{2}\left(-1+4 b^{2}+n+2 n b^{2}\right) s_{0}^{k} s_{k}+6\left(1+2 b^{2}\right)^{3}\left(3+2 b^{2}\right) \beta s^{j}{ }_{k} s^{k}{ }_{j}+4\left(5+4 b^{2}\right)\left(1+2 b^{2}\right)^{3} \beta \sigma \\
& +12\left(1+2 b^{2}\right)^{2}\left(5+4 b^{2}\right) \beta s^{k} s_{k} ; \\
t_{14} & =-\left(1+2 b^{2}\right)^{4} s_{k}{ }_{k} s^{k}{ }_{j}-\left(1+2 b^{2}\right)^{4} \sigma-4\left(1+2 b^{2}\right)^{3} s^{k} s_{k} .
\end{aligned}
$$

\section{REFERENCES}

[1] T.Aikou, M.Hashiguchi and K.Yamaguchi, On Matsumoto's Finsler space with time measure, Rep. Fac. Sci. Kagoshima Univ. (Math. Phys. Chem), 23(1990), 1-12.

[2] H.Akbar-Zadeh, Generalized Einstein manifolds, J. Geom. and Phys., 17(1995), 342-380.

[3] D.Bao and C.Robles, Ricci and flag curvatures in Finsler geometry, in "A Sampler of Finsler Geometry", MSRI series 50, Camb. Univ. Press, 2004, 197-259.

[4] X.Cheng, Z.Shen and Y.Tian, A Class of Einstein $(\alpha, \beta)$-metrics, Israel Journal of Mathematics, accepted.

[5] S.S.Chern and Z.Shen., Riemann-Finsler geometry, World Scientific, 2005.

[6] B.Li, Projectively flat Matsumoto metric and its approximation, Acta Mathematica Scientia 2007, 27B(4), 781-789.

[7] M.Matsumoto, A slope of a mountain is a Finsler surface with respect ot time measure, J. Math. Kyoto Univ., 29(1989), 17-25.

[8] H.S.Park, I.Y.Lee and C.K.Park, Finsler space with the general approximate Matsumoto metric, Indian J. pure and appl. Math., 34(1)(2002), 59-77.

[9] M.Rafie-Rad and B.,Rezaei, Matsumoto metrics of constant flag curvature are trivial, Results. Math., Online First, 2011, Springer Basel AG, DOI 10.1007/s00025-011-0210-1.

[10] M.Rafie-Rad and B.,Rezaei, On Einstein Matsumoto metrics, Nonlinear Anal. Real World Appl., Vol. 13, Issue 2, 2012, 882-886.

[11] B.Rezaei, A.Razavi and N.Sadeghzadeh, ON EINSTEIN $(\alpha, \beta)$-METRICS*, Iranian Journal of Science Technology, Transaction A. Vol. 31. No. A4 Printed in The Islamic Republic of Iran, 2007.

[12] X.Zhang. and Yi.Shen., On Einstein Kropina metrics, to appear in Differential geometry and its Applications.

[13] L.Zhou, A local classification of a class of $(\alpha, \beta)$-metrics with constant flag curvature, Differential geometry and its Applications, 28(2010), 170-193.

[14] S.Bácsó, X.Cheng and Z.Shen., Curvature properties of $(\alpha, \beta)$-metrics, Advanced Studies in Pure Mathematics, Math. Soc. of Japan, 48(2007), 73-110.

\section{Yi-Bing Shen}

Center of Math. Science,

Yuqun Campus, Zhejiang University,

Hangzhou 310027, China,

Email: yibingshen@zju.edu.cn

Xiaoling Zhang

Department of Mathematics,

Zhejiang University,

Hangzhou 310027, China,

College of Mathematics and Systems Science,

Xinjiang University,

Urumqi 830046, China,

Email:xlzhang@ymail.com 